\title{
Nódulos linfáticos parietales en inspección rutinaria de caprinos
}

\author{
Bode, F.F. ${ }^{1}$; Fernández, J.A.'; Resoagli, J.M.'; Villordo, G.I. ${ }^{1}$ Polej, E. ${ }^{2}$ \\ ${ }^{1}$ Cátedra de Anatomía I, ${ }^{2}$ Cátedra de Bromatología, Fac.Cs.Veterinarias, UNNE, \\ Cabral 2139, Corrientes, Argentina. E-mail: alberto.fernandez@usal.edu.ar
}

\begin{abstract}
Resumen
Bode, F.F.; Fernández, J.A.; Resoagli, J.M.; Villordo, G.I.; Polej, E.: Nódulos linfáticos parietales en inspección rutinaria de caprinos. Rev. vet. 29: 1, 61-64, 2018. El presente trabajo tuvo como objetivo identificar y describir los nodulos linfaticos de los caprinos, para establecer criterios sanitarios en el control de carne destinada al consumo. Se abordó la situación, forma y tamaño de los nódulos linfáticos (NL). Se utilizaron seis ejemplares adultos. Previa anestesia general, se realizó la eutanasia e inyección de tinta china a fin de que el drenaje linfático fuera visible en la disección. Se observó que el NL cervical superficial o pre escapular, de forma elíptica, se situó en el borde craneal de la escápula. Los NL sub-ilíacos o pre-femorales, alargados, se situaron en craneal del músculo tensor de la fascia lata, equidistantes de la patela y el tubérculo coxal. El NL retro mamario, ovalado, se localizó en la región inguinal, rodeado de abundante cantidad de grasa. El NL poplíteo, de forma ovoide, se ubicó entre los músculos bíceps femoral y el contorno craneal del músculo semitendinoso. El NL prepectoral, costo cervical o falso ganglio del inspector, de forma alargada, se halló a lo largo de la superficie ventral de la tráquea. El NL ilíaco medio, con forma de coma, se ubicó cerca de la bifurcación de la aorta abdominal. El NL ilíaco lateral, redondeado, se situó en lateral del origen de los vasos ilíacos externos. A los NL isquiáticos, de forma ovoide, se los localizó en la incisura isquiática mayor, en craneal de la cresta isquiática. Los resultados difieren de los descriptos en esta especie por otros autores, en cuanto al número y tamaño de determinados linfocentros. El hallazgo permitirá adecuar los sistemas de vigilancia durante la inspección de rutina en la playa de faena y mejorar la calidad de los alimentos que llegan al consumidor.
\end{abstract}

Palabras clave: caprino, nódulos linfáticos parietales, bromatología.

\begin{abstract}
Bode, F.F.; Fernández, J.A.; Resoagli, J.M.; Villordo, G.I.; Polej, E.: Parietal lymph nodes on routine inspection in goats. Rev. vet. 29:1, 61-64, 2018. The present work is part of a project whose objective was to describe the lymphatic nodules of goats, for the application of sanitary criteria in the control of meat destined for consumption. This report describes the status, shape and size of lymph nodes (NL). Six adult specimens were used. After general anesthesia, euthanasia was performed. Subsequent to this, chinese ink is injected, so lymphatic drainage was visible in the dissection. It was observed that the superficial cervical or scapular $\mathrm{NL}$, elliptic, is located at the cranial border of the scapula. The elongated NL sub-iliacus or pre femoral, were located in cranial of the tensor muscle of the fascia lata, equidistant of the patella and the coxal tuber. The NL retro mammary had oval shape and it was located in the inguinal region, surrounded by abundant fat. The popliteal NL had ovoid form, was located between the biceps femoris muscles and cranial contour of the semitendinosus muscle. The prepectoral NL, cervical cost or false lymph node of the inspector, elongated, was found along the ventral surface of the trachea. The middle iliac NL, had coma-shaped, it was located near the bifurcation of the abdominal aorta. The lateral iliac NL had a circular shaperounded, it was located lateral to the origin of the external iliac vessels. The sciatic nodes had ovoid shape, it were located in the major ischial incision, in the cranial of the ischial ridge. From the analysis of the results it was established that the external parietal lymphatic structures in routine inspection in goats, differ from those described in this species by other authors, in reference to the number and size in certain lymphocentres, this will allow to adapt the surveillance systems during the health inspection and will improve the quality of the food that reaches the consumer.
\end{abstract}

Key words: goat, parietal lymph nodes, bromatology. 


\section{INTRODUCCIÓN}

Según estimaciones oficiales, en Argentina existen alrededor de 4 millones de caprinos, la mayoría de ellos de tipo criollo, que sirven de soporte económico a 50.000 pequeños productores de escasos recursos y bajo nivel sociocultural ${ }^{9}$. En 2005, la región nordeste albergaba el $14,5 \%$ del stock caprino total del país. Las existencias caprinas en cabezas/provincia se distribuían de la siguiente manera: Chaco 342.457; Formosa 217.846; Corrientes 22.499 y Misiones 2.677 cabezas.

Tal producción de carne caprina está orientada principalmente a la obtención del cabrito mamón o chivitos para consumo, que constituyen el producto tradicionalmente comercializado. Los cabritos, que se faenan a una edad de 40-50 días y con un peso vivo aproximado de 7-9 $\mathrm{kg}^{1}$, contienen bajos niveles de grasa y colesterol intramuscular ${ }^{3}$, atributos importantes para que sean considerados como alimentos de alta calidad nutricional.

Otro producto que reviste especial importancia para la actividad de exportación regional es la producción de cabras de refugo, lo que confiere mayor atención sanitaria a la hora de la inspección para el diagnóstico de enfermedades de alto riesgo sanitario como la tuberculosis, por lo que se torna indispensable el conocimiento de la topografía ganglionar para un correcto diagnóstico y posterior aplicación de los diferentes criterios sanitarios específicos.

Desde el punto de vista del consumo de carne de animales producidos en distintos sistemas, existe riesgo sanitario debido a la falta parcial o total de control bromatológico, ya que no se cumple con la garantía de la inocuidad de un producto, es decir la reducción de los riesgos que conlleva su consumo ${ }^{2}$.

La preocupación de la comunidad en relación a la inocuidad de los alimentos aumentó en los últimos años. De acuerdo a la Organización Mundial de la Salud, las enfermedades transmitidas por alimentos constituyen una importante causa de morbilidad y mortalidad, ya que afectan a personas de todas las edades, pero sobre todo a niños menores de cinco años ${ }^{12}$. Por ello, resulta necesario incrementar la inspección sanitaria de las carnes destinadas al consumo.

Si bien en el bovino existe amplia información sobre las estructuras linfáticas, dadas las exigencias bromatológicas sobre inspección sistemática de nódulos linfáticos, no ocurre así con la especie caprina, posiblemente porque en la práctica general se intenta relacionar morfológica y funcionalmente ambas especies.

La localización de los ganglios linfáticos en la especie caprina recuerda en líneas generales a la del vacuno, sin embargo se observa que el sistema linfático de las cabras no se ha estudiado adecuadamente ${ }^{4,5,6,11}$ . Los ganglios y los vasos linfáticos pueden modificar sus características normales en distintas enfermedades infecciosas ${ }^{8}$.

Según la Nómina Anatómica Veterinaria, un linfocentro es un nódulo linfático o un grupo de nódulos linfáticos que se presentan en la misma región del cuerpo y reciben vasos aferentes desde la misma zona en la mayoría de los mamíferos domésticos. Por su parte, linfonódulo es una pequeña masa sólida de tejido linfático, carente de luz ${ }^{7}$.

La presencia de los principales linfocentros es constante en los distintos mamíferos destinados al consumo, pero como se mencionó anteriormente, pueden existir variaciones en cuanto al tamaño, número, color y posición, entre e incluso dentro de una misma especie, por lo que es indispensable realizar un mapeo topográfico de los mismos para facilitar la exploración, de importancia indiscutible para los técnicos sanitarios.

En la regulación sanitaria argentina, el Reglamento de Inspección de Productos, Subproductos y Derivados de Origen Animal (Decreto 4238/68) no contiene especificaciones en cuanto a la inspección obligatoria de ganglios linfáticos parietales y viscerales en los caprinos ${ }^{10}$.

El objetivo de este trabajo fue identificar y describir la situación, forma y tamaño de los diferentes nódulos linfáticos (NL) de inspección de rutina en la playa de faena de la especie caprina, así como detallar los vasos aferentes y eferentes de cada uno de los linfocentros, datos de suma importancia para definir el destino de la res.

\section{MATERIAL Y MÉTODOS}

Se emplearon seis ejemplares adultos de la especie caprina, de ambos sexos, con un peso promedio de alrededor de $50 \mathrm{~kg}$. La eutanasia se realizó previa anestesia general con acepromacina $(0,1 \mathrm{mg} / \mathrm{kg})$ y ketamina $(15 \mathrm{mg} / \mathrm{Kg})$ vía endovenosa.

Posteriormente, se inyectó tinta china azul con aguja $25 / 8$ y jeringa de $3 \mathrm{ml}$, en la piel de los espacios interdigitales de los miembros torácicos y pelvianos, parte caudal del cuello, pared costal, pared abdominal y zona perineal, a fin de que el drenaje linfático fuera visible en la disección.

Los especímenes se conservaron en cámara fría hasta su procesamiento. Para el estudio topográfico de los linfocentros, los NL se clasificaron en parietales o musculares y viscerales. A los NL parietales se los dividió -de acuerdo a su ubicación- en cervicales, torácicos, pelvianos y viscerales de las cavidades torácica, abdominal y pelviana.

Los datos fueron registrados fotográficamente con cámara Nikon L 110. Las mediciones se realizaron con calibre milimetrado y los valores numéricos fueron procesados estadísticamente para obtener media aritmética y desviación estándar.

\section{RESULTADOS Y DISCUSIÓN}

Con el animal en decúbito lateral se observó que el NL cervical superficial o pre- escapular asumió una forma elíptica, de $3,88 \pm 0,51 \mathrm{~cm}$ de largo por 1,17 \pm $0,21 \mathrm{~cm}$ de ancho, ubicándose en el borde craneal de la escápula, proximal a la articulación del encuentro. Los 


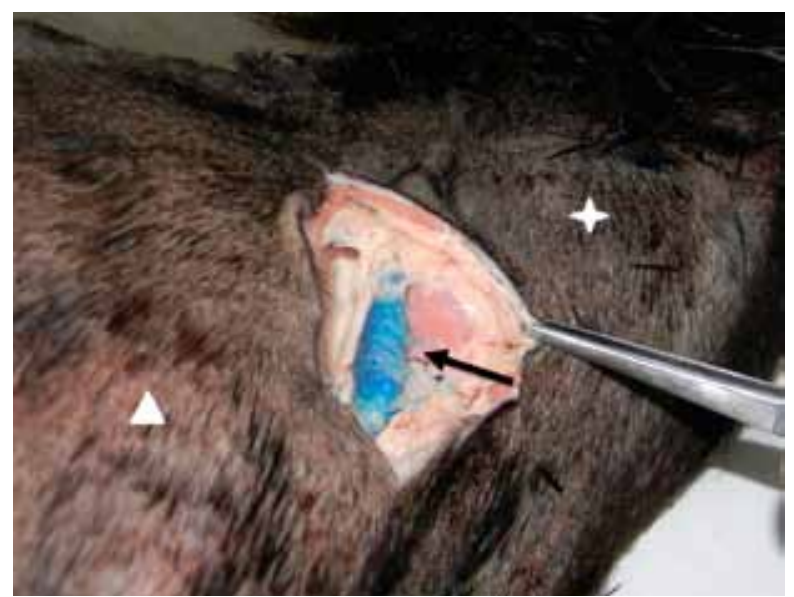

Figura 1. NL pre-escapular (flecha). Región cervical (estrella). Región escapular (triángulo).

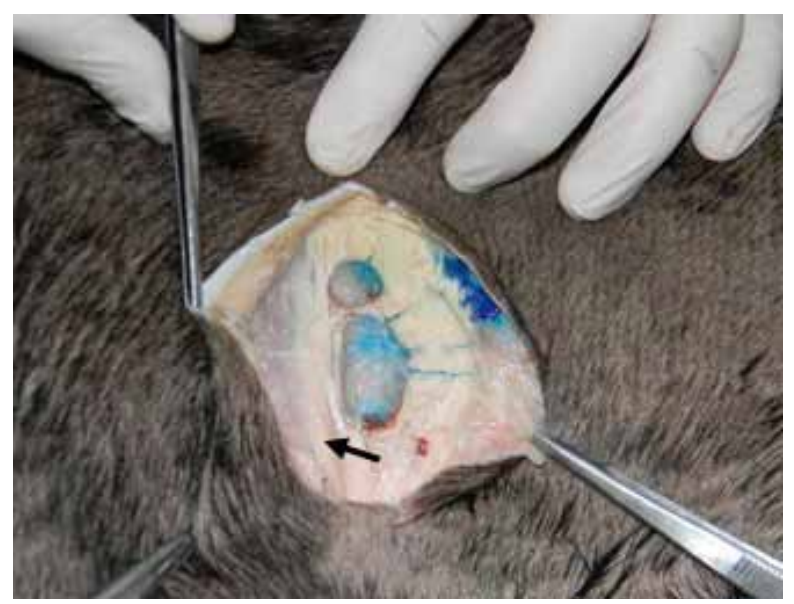

Figura 2. NL pre-femoral derecho. Nótese el drenaje linfático a la derecha del NL de mayor tamaño. Músculo tensor de la fascia lata (flecha).

vasos aferentes provinieron de la piel, espacio interdigital y miembro torácico; los eferentes drenaron en el tronco traqueal (Figura 1).

En el miembro izquierdo, los linfonódulos sub-ilíacos o pre-femorales de la región pelviana, constituyeron un nódulo de forma alargada, aplanado lateralmente, de 3,02 $\pm 0,64 \mathrm{~cm}$ de largo por 0,98 $\pm 0,18 \mathrm{~cm}$ de ancho. En el miembro derecho se observaron dos nódulos, uno de forma alargada de 2,90 $\pm 0,54 \mathrm{~cm}$ de largo por $0,80 \pm 0,18 \mathrm{~cm}$ de ancho, y otro de forma circular, de $1.1 \pm 0,25 \mathrm{~cm}$ de diámetro, que se ubicaron en craneal del músculo tensor de la fascia lata, equidistantes de la patela y el tubérculo coxal. Los vasos aferentes procedieron de la región abdominal, inguinal y perineal, los vasos eferentes descargaron en los linfáticos ilíacos medios (Figura 2).

Los linfonódulos retro-mamarios en la hembra (inguinal superficial en el macho) fueron dos nódulos linfáticos ubicados en la región inguinal, rodeados de gran cantidad de grasa, de forma oval, con 2,55 $\pm 0,69$ $\mathrm{cm}$ de largo y $1,40 \pm 0,38 \mathrm{~cm}$ de ancho y otro elíptico de $1,1 \mathrm{~cm}$ de largo por $0,8 \mathrm{~cm}$ de ancho, que se situaron dorso caudalmente a la glándula mamaria. Los vasos aferentes provinieron de la glándula mamaria, piel y re-

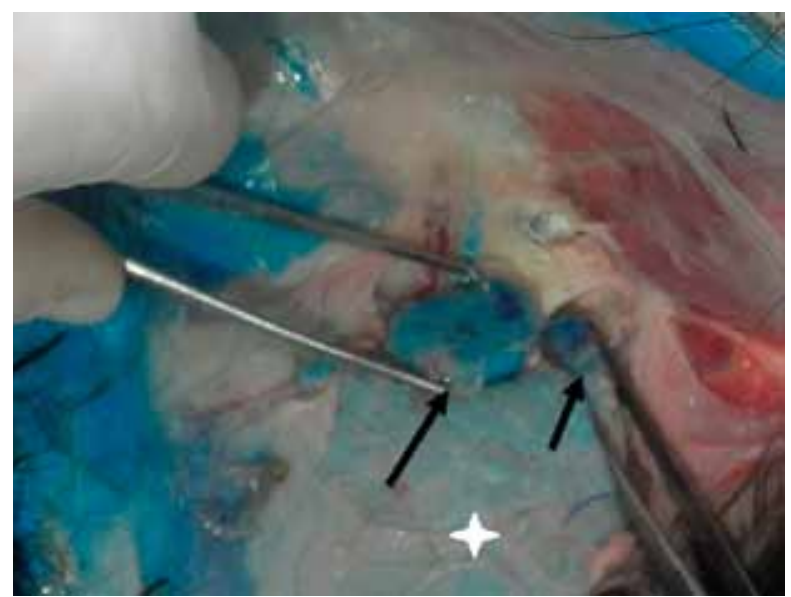

Figura 3. NL retromamario (flechas). Glándula mamaria (estrella).

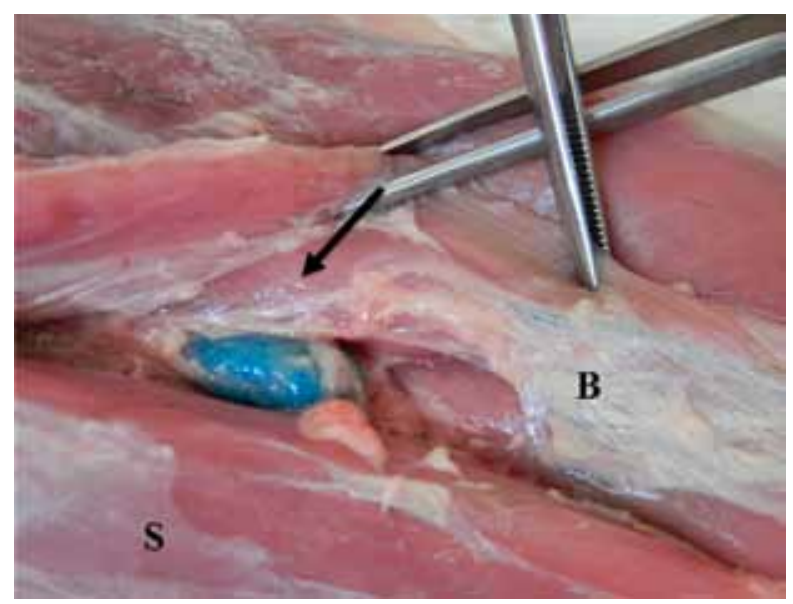

Figura 4. NL poplíteo (flecha). Músculos biceps femoral (B) y semitendinoso (S).

gión femoral medial, los vasos eferentes drenaron en los nódulos ilíacos mediales o el tronco lumbar (Figura 3).

El linfonódulo poplíteo de la región pelviana, de forma ovoide, con 1,88 $\pm 0,30 \mathrm{~cm}$ de largo por 1,03 \pm $0,27 \mathrm{~cm}$ de espesor, se ubicó entre los músculos bíceps femoral y el contorno craneal del músculo semitendinoso. Los vasos aferentes drenaron en la parte distal del miembro pelviano y los vasos eferentes en los nódulos iliacos laterales, mediales e isquiáticos (Figura 4).

El NL prepectoral, costo cervical o falso ganglio del inspector, alargado, con 2,27 $\pm 0,25 \mathrm{~cm}$ de largo por $0,67 \pm 0,06 \mathrm{~cm}$ de ancho, se situó en la cara ventral de la tráquea, en craneal de la primera costilla. Las aferencias procedieron de los músculos circundantes a la tráquea, esófago y del tronco traqueal derecho. Los vasos eferentes drenaron en el conducto torácico y en NL axilares de la primera costilla.

El linfonódulo ilíaco medio, con forma de coma, midió 2,60 $\pm 0,86 \mathrm{~cm}$ de largo por $1,08 \pm 0,88 \mathrm{~cm}$ de ancho, ubicándose en la entrada de la cavidad pelviana, a nivel de la bifurcación de la aorta abdominal, en el ángulo que existe entre los vasos ilíacos externos, la aorta abdominal y la vena cava caudal. Recibieron aferencias de los órganos que se alojan en la cavidad 
pelviana y de los NL poplíteos, isquiáticos, ilíacos laterales, ano rectales, subilíacos e inguinal superficial en el macho o retromamarios en la hembra. Las eferencias descargaron en el tronco lumbar.

Se verificó la presencia de uno a dos linfonódulos ilíacos laterales en la cavidad pelviana, redondeados, de $2,85 \pm 0,83 \mathrm{~cm}$ de largo por $1,40 \pm 0,29 \mathrm{~cm}$ de ancho. Estos nódulos se situaron en lateral del origen de los vasos ilíacos externos y recibieron aferencias de los órganos de la cavidad pelviana, de los músculos y articulaciones del miembro pelviano, así como de los NL inguinal superficial o retromamario, isquiático y pre-femoral. Los vasos eferentes drenaron en el tronco lumbar, cisterna del quilo, nódulos linfáticos ilíacos medios y aórticos lumbares.

En la región pelviana, los NL isquiáticos, de forma ovoide, con 1,27 $\pm 0,60 \mathrm{~cm}$ de largo por $0,72 \pm 0,23 \mathrm{~cm}$ de ancho, se localizaron en la incisura isquiática mayor, en craneal de la cresta isquiática. Los vasos aferentes provinieron de la piel, articulaciones de la cadera, músculos de la región glútea, de la cola y del ano. Los vasos eferentes drenaron en los nódulos linfáticos ilíacos y lumbares.

Las estructuras linfáticas de inspección rutinaria en la especie caprina, no difieren mayormente de las descriptas en la especie bovina ${ }^{4,5,11}$, resultando accesibles al momento de realizar la inspección post-mortem en la playa de faena, mediante las técnicas de observación macroscópica y palpación de las mismas. No obstante, en los caprinos se destacan variables en la presencia, número y tamaño de determinados linfocentros, lo cual requerirá adecuar las inspecciones durante el examen de rutina, y así mejorar la calidad de los alimentos que llegan al consumidor.

\section{REFERENCIAS}

1. Dayenoff P, Bolaño M, Aguirre E, Giovanardi F. 1997. Crecimiento post-destete del cabrito tipo criollo regional. Rev Arg Prod Anim 17: 1-27
2. FAO/OMS. 1997. Gestión de riesgos e inocuidad de los alimentos (Estudio FAO: Alimentación y Nutrición). http://www.fao.org/docrep/w4982s/w4982s00.htm

3. Garriz C. 1994. Evaluación integral de calidad de res y carne de cabritos criollos argentinos. Anales ler Curso Integral de Lechería Caprina (INTA-CICV), Castelar, Buenos Aires.

4. Getty R. 1982. Anatomía de los Animales Domésticos, Cap. 34: sistema linfático de los rumiantes, Parte III, Caprinos, $5^{\circ}$ ed., Salvat, Barcelona, p. 1134-1176.

5. König H, Liebich H. 2005. Anatomía de los animales domésticos. II: Órganos, sistema circulatorio y sistema nervioso. Ed. Médica Panamericana, 2a ed., Buenos Aires, $400 \mathrm{p}$.

6. Mayer HF. 1984. Bromatología, Higiene y Control de Alimentos, Ed. Impresiones UNNE, Resistencia (Chaco, Argentina), p 45-46.

7. Nómina Anatómica Veterinaria. 2012, $5^{\text {th }}$ ed., Editorial Committee WAVA, Hannover (Germany), $166 \mathrm{p}$.

8. Rosenberger G. 1994. Exploración Clínica de los Bovinos, $3^{\circ}$ ed., Hemisferio Sur, BuenosAires, Argentina, 59 p.

9. SAGPYA. 2005. Existencias de ganado caprino. Publ. Dirección de Ganadería de la. Secretaría de Agricultura, Ganadería, Pesca y Alimentación de la Nación, 23 p.

10. SENASA. 1968. Reglamento de inspección de productos, subproductos y derivados de origen animal (Decreto Ley 4238/68), Buenos Aires, Argentina). https://vieja-web.senasa.gov.ar/Archivos/File/File753-capitulos.pdf

11. Sisson S, Grossman JD. 1963. Anatomía de los Animales Domésticos, $4^{\circ}$ ed., Salvat, Barcelona.

12. Organización Mundial de la Salud. 2015. Estimación de la carga mundial de las enfermedades de transmisión alimentaria. http://apps.who.int/iris/bitstream/10665/199350/ 1/9789241565165_eng.pdf 